IdeAs

Idées d'Amériques

$10 \mid 2017$

États-Unis / Cuba : une nouvelle donne?

\title{
La médiation du pape François entre La Havane et Washington : rupture ou continuité dans la diplomatie pontificale?
}

Pope's Francis mediating work between Havana and Washington, change or continuity in papal diplomacy?

La mediación de papa Francisco entre la Habana y Washington: ¿ruptura o continuidad en la diplomacia pontificia?

\section{Marie Gayte}

\section{OpenEdition}

Journals

Édition électronique

URL : https://journals.openedition.org/ideas/2191

DOI : 10.4000/ideas. 2191

ISSN : $1950-570$

Éditeur

Institut des Amériques

Référence électronique

Marie Gayte, «La médiation du pape François entre La Havane et Washington : rupture ou continuité dans la diplomatie pontificale? », IdeAs [En ligne], 10 | 2017, mis en ligne le 19 décembre 2017, consulté le 18 octobre 2022. URL : http://journals.openedition.org/ideas/2191 ; DOl : https://doi.org/ $10.4000 /$ ideas. 2191

Ce document a été généré automatiquement le 18 octobre 2022

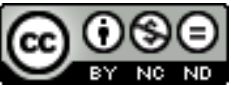

Creative Commons - Attribution - Pas d'Utilisation Commerciale - Pas de Modification 4.0 International - CC BY-NC-ND 4.0

https://creativecommons.org/licenses/by-nc-nd/4.0/ 


\title{
La médiation du pape François entre La Havane et Washington : rupture ou continuité dans la diplomatie pontificale?
}

\author{
Pope's Francis mediating work between Havana and Washington, change or \\ continuity in papal diplomacy? \\ La mediación de papa Francisco entre la Habana y Washington: ¿ruptura o \\ continuidad en la diplomacia pontificia?
}

Marie Gayte

\section{Introduction}

Le Saint-Siège compte à son actif une longue tradition de médiation entre États, notamment depuis le pontificat de Léon XIII (1878-1903). Outre la volonté pacificatrice du pontife, le siège de l'Église catholique, qui a perdu en 1870 presque toute assise temporelle - hormis les quelques hectares de la Cité du Vatican - connaît au tournant du siècle dernier une évolution auprès de l'opinion, qui voit de plus en plus en lui une institution impartiale, car détachée des contingences territoriales, et de ce fait capable d'assurer une fonction médiatrice entre États (Ticchi J.M., 1993: 572). Depuis lors, le Saint-Siège a assuré de nombreuses médiations (Cardinale I., 1962 : 46 ; Noé J.-B., 2015 : 34-35), certaines relatives à des États non-catholiques, mais elle est intervenue tout particulièrement pour régler des différends territoriaux impliquant des pays d'Amérique latine (Barberini G., 2003: 178)1. Cette tradition perdure jusqu'à aujourd'hui puisque très récemment, le Saint-Siège a été sollicité pour débloquer l'impasse des négociations en Colombie entre le gouvernement de Juan Manuel Santos et les FARC (Ordaz P., 2015), ainsi que pour tenter de trouver au Venezuela une solution au conflit entre le régime de Nicolas Maduro et l'opposition dans le cadre de la 
médiation proposée par les anciens chefs d'État José Luis Rodríguez Zapatero, Martín Torrijos, Ernesto Samper et Leonel Fernandez (Castro M., 2016 ; Infobae, 2017 ; Falasca S., 2017).

2 Cette disposition à proposer ses bons offices se double d'une réelle compétence diplomatique, fruit d'une présence quasi universelle ainsi que de l'existence d'un corps diplomatique professionnel formé à l'Académie pontificale ecclésiastique. En 2014, 180 États étaient représentés à la cour de Rome (Noé J.-B., 2015 : 83). La présence d'évêques, de prêtres et d'ordres religieux à travers le monde assure de plus des relais d'information - voire d'influence - non négligeables, qui permettent au Saint-Siège d'avoir une connaissance relativement fine des situations locales.

3 Les États-Unis en sont bien conscients, ayant eux-mêmes recouru aux bons offices romains à plusieurs reprises au sujet de nations d'Amérique latine. Ainsi, à l'occasion de la crise des missiles de Cuba de 1962, à la demande du président américain, le pape Jean XXIII avait exhorté J. Kennedy et N. Khrouchtchev à faire baisser les tensions (Di Nolfo E. 1993 :109-122). De même, en 1978, l'administration Carter avait sollicité JeanPaul II pour chercher une solution au conflit opposant le Chili et l'Argentine sur la souveraineté du canal de Beagle. Ce dernier nomma un diplomate chevronné pour assurer une médiation couronnée de succès (Day M., 1981 : 20).

4 C'est donc dans ce contexte particulier que s'inscrit la nouvelle, annoncée lors des allocutions télévisées respectives de Barack Obama et Raul Castro, le 17 décembre 2014, selon laquelle le pape François aurait assuré une médiation déterminante dans le rapprochement cubano-américain. On apprend ainsi de la bouche du président étatsunien que le pape lui a demandé ainsi qu'à Raul Castro de résoudre le cas d'Alan Gross et des « agents cubains détenus aux États-Unis depuis quinze ans » (Obama B., 2014).

La secrétairerie d'État du Saint-Siège publie à son tour un communiqué dans lequel elle se félicite de la décision et évoque, outre l'envoi de lettres exhortant les deux dirigeants à résoudre la question des prisonniers «afin de permettre le début d'une nouvelle phase dans les relations entre les deux pays", la tenue d'une rencontre entre négociateurs américains et cubains au Vatican en octobre 2014 et le rôle de «bons offices de la part du Vatican pour permettre un dialogue constructif sur des questions délicates, débouchant sur une solution acceptable par les deux parties» (McElwee J., 2014).

5 Cette annonce intervient alors que règne au Vatican depuis dix-huit mois le premier pape d'Amérique latine, Jorge Mario Bergoglio, pape qui semble à plusieurs égards incarner une rupture d'avec son prédécesseur, Benoît XVI. Elle se produit également alors que les États-Unis semblent avoir changé de stratégie envers Cuba. Après avoir tenté d'obtenir du Vatican depuis une quinzaine d'années que ce dernier œuvre à leurs côtés pour une transition démocratique, ils sont passés sous la présidence de Barack Obama à une attitude plus conciliante et plus favorable (au moins à moyen terme) au maintien d'une certaine forme de statu quo dans l'île des Caraïbes. L'annonce du rapprochement se produit également à un moment où Cuba souffre des effets d'une crise économique due notamment à la baisse du soutien financier vénézuélien et du manque de résultats des réformes impulsées par Raul Castro depuis 2006.

Cet article se propose de montrer comment un concours de circonstances inédit a permis cette médiation pontificale, après l'existence de divergences entre les différents acteurs rendant jusque lors impossible une telle intervention. Il s'agit également de démontrer que ce concours de circonstances ne doit pas occulter le fait que l'action du 
pape François, si elle s'inscrit dans une longue tradition de bons offices dans la région, n'est pas le reflet d'une "rupture » dans la diplomatie vaticane. Cette dernière reste avant tout motivée par des principes - sauvegarde de l'institution ecclésiale et des fidèles, choix du dialogue plutôt que de la confrontation - qui sont ceux qui animent l'action du Saint-Siège depuis que ce dernier joue un rôle important sur la scène internationale - depuis le pontificat de Léon XIII jusqu'à ceux des deux prédécesseurs immédiats de François, Jean-Paul II et Benoît XVI.

\section{De profondes divergences entre Washington et le Saint-Siège sur le dossier cubain}

\section{L'Église catholique, de paria à « interlocuteur privilégié » du régime castriste}

7 La révolution cubaine a pour le moins distendu les liens entre La Havane avec Rome. Dans les trois années qui suivirent la révolution de 1959, l'emprisonnement dans des camps de travail et l'expulsion de nombreux prêtres et de religieux, la fermeture des séminaires, la nationalisation des écoles catholiques et l'imposition de l'athéisme furent autant de coups semblant parfois mettre en péril jusqu'à l'existence même de l'Église sur l'île (Holbrook J., 2010 : 264-275 ; Alonso A., 2009 : 290, 2002 : ch. 1).

8 Pour distendus qu'ils aient été, les liens n'ont cependant jamais été complètement rompus, signalant dès les origines une différence d'approche entre le Saint-Siège et les États-Unis. Le 3 février 1962, le nouvel ambassadeur cubain présentait ses lettres de créance à Jean XXIII, alors qu'au même moment le président Kennedy annonçait des sanctions totales contre l'île et que Cuba avait été exclue de l'Organisation des États américains au sommet de Punta del Este trois jours auparavant (Dupuy A., $1980: 228$ ).

En dépit de toutes les vexations qu'a dû subir l'Église cubaine, le Vatican n'a jamais suspendu ses relations diplomatiques avec la capitale cubaine, établies en 1935, même s'il y fut longtemps représenté par un chargé d'affaires et non par un nonce apostolique (Quigley T., 2003: 95). Ce choix de maintenir les lignes de communication ouvertes a parfois été perçu comme un aspect contradictoire de la diplomatie du Saint-Siège, mais en agissant de la sorte il s'agissait avant tout pour Rome de ne pas abandonner à leur sort les fidèles et les institutions catholiques de l'île (le Saint-Siège par le biais de sa publication l'Osservatore Romano eut tout de même à cœur de démentir certaines affirmations de Fidel Castro concernant l'absence d'hostilité de son régime envers l'Église). Cette attitude de compromis et de dialogue de la part de Rome - et de plus en plus de la part de l'épiscopat cubain également - n'est pas sans rappeler l'Ostpolitik menée par Mgr Casaroli pendant le pontificat de Paul VI vis-à-vis de la Yougoslavie de Tito ou de la Hongrie $^{2}$, Ostpolitik qui fut, comme à Cuba, critiquée par certains catholiques comme une forme de trahison. Cette ouverture au dialogue de la part de Rome permit à l'Église à Cuba de connaître certaines avancées. Les évêques cubains purent assister au concile Vatican II ; quelques prêtres furent autorisés à s'installer sur l'île, prêtres dont la pénurie risquait de porter un coup fatal à la survie même de l'institution, et en 1974 Mgr Casaroli se rendit à Cuba pour une visite «strictement ecclésiale », mais à l'occasion de laquelle il put s'entretenir avec Fidel Castro (Dupuy A., 1980 : 229-230). Pour autant, l'attitude cubaine fut parfois proche de celle des régimes est-européens - souvent peu disposés à tenir les promesses faites à Rome. Fidel Castro 
supprima ainsi le jour férié de Noël en 1969. Le Vatican n'eut pour autant de cesse de condamner l'embargo américain sur l'île pour ce qu'il entraînait de difficultés pour la population. Du côté des évêques, après une période de fortes tensions, se produisit une période qualifiée de " période de silence» (Hurtado R.F. et T. Henken, $2013: 186)$. Ce silence fut néanmoins rompu par deux lettres pastorales de 1969 dénonçant l'embargo et appelant à la réconciliation entre croyants et non-croyants (Alonso A. 2009 : 306).

L'approche adoptée par le Vatican à Cuba est l'illustration d'une des principales priorités de sa diplomatie. Comme le rappelle Giovanni Barberini, «[1]e Saint-Siège opère comme un sujet qui entend faire valoir ses propres intérêts spécifiques, ainsi que les intérêts généraux de la communauté internationale, considérés du point de vue humanitaire, moral et spirituel » (Barberini G., 2003: 88). Si la promotion de la paix entre les peuples fait partie des missions de l'Église - notamment depuis la publication de la constitution pastorale Gaudium et Spes issue du concile Vatican II (Barberini G., 2003 : 90) - de même que la défense de la dignité humaine et des droits humains, comme stipulé dans l'encyclique Pacem in Terris de Jean XXIII (Barberini G., 2003 : 102 ; 109), Barberini rappelle néanmoins que :

[1]e Saint-Siège agit avant tout dans l'intérêt de l'Église universelle, qui passe par ceux des Églises particulières, ainsi que dans l'intérêt des institutions catholiques locales dans les différentes parties du monde. [...] Ces intérêts comprennent évidemment la liberté et l'autonomie des institutions ecclésiastiques, les droits et les libertés des fidèles, de même que les conditions de développement et de croissance de la confession catholique en général [...] (Barberini G., $2003: 88$ ).

11 C'est dans cet esprit que, dès les années 1980, Jean-Paul II envisage un voyage à Cuba, pour des raisons de sauvegarde de l'Église, qui s'y trouve en fâcheuse posture. Cette dernière ne fut jamais très forte, notamment de par le manque de prêtres et du fait que les rares ecclésiastiques présents sur l'île étaient espagnols, c'est-à-dire de la nationalité de l'occupant jusqu'en 1898, d'où un certain anticléricalisme (Alonso A., 2009: 289). L'Église fut en outre étroitement associée aux classes dirigeantes. La pratique religieuse était relativement faible avant même la Révolution, cette dernière ne contribuant évidemment pas à la renforcer (Létrillart P., 2005 : 11-12). Il y avait donc pour le pontife polonais urgence à insuffler une dose de ferveur catholique chez les Cubains, surtout que l'île pendant la "période spéciale " montrait les signes d'une forme de renouveau spirituel, un renouveau dont profitaient surtout la religion populaire et les Églises pentecôtistes et très peu l'Église catholique pour les raisons historiques mentionnées supra (Alonso A., 2009 : 292-293 ; Hurtado R.F. et T. Henken, $2013: 188)$.

12 Les Américains pratiquent alors de leur côté une politique d'isolement maximal de lîle, dans l'espoir de précipiter la chute du régime, et dans ce contexte déploient une grande énergie à y empêcher toute présence internationale. Ils sont de ce fait rétifs à l'idée d'un tel voyage quand le Vatican en émet l'idée en 1985 (Van Heuven M., 1985). L'éventualité d'un déplacement pontifical sur l'île est à nouveau évoquée en 1989 puis en 1992. A chaque fois, les Américains font savoir au Saint-Père qu'ils jugent un tel déplacement «non opportun" (Melady T., 1994: 79). La volonté de Jean-Paul II de se rendre à Cuba est d'autant plus mal comprise que Washington tend à voir dans le pontife polonais un allié inconditionnel dans la lutte idéologique contre Moscou (Van Heuven M., 1985).

13 Les années 1980 sont le théâtre d'une certaine forme de "réconciliation » entre Cuba et le Saint-Siège, avec la publication en 1985 d'un ouvrage d'entretiens avec Fidel Castro 
par le dominicain brésilien Frei Betto intitulé Fidel et la religion. Le régime cubain a ses raisons d'envoyer des signaux plus positifs en direction de l'Église au cours de cette décennie, voire à se montrer disposé à accueillir le souverain-pontife. A partir de l'accession de Mikhaïl Gorbatchev en 1985 au Kremlin, une certaine distance se creuse entre La Havane et Moscou. Les évêques commencent pour leur part à mettre en œuvre les recommandations de la conférence de Puebla de 1979 en faveur d'une option préférentielle pour les pauvres. En 1986, l'Église cubaine adopte une attitude plus pragmatique envers le régime (Alonso A., 2009 : 290). En 1989 Fidel Castro invite une fois de plus Jean-Paul II à se rendre sur l'île (Alonso A., 2009 : 297). Les bouleversements à l'Est incitent le pape à un certain attentisme, alors qu'ils rendent pour Fidel Castro un rapprochement avec l'Église catholique d'autant plus urgent que, outre le fait qu'une visite pontificale soit susceptible de contribuer à briser l'isolement de Cuba, l'Église pourrait fournir sur l'île, en pleine crise économique avec le tarissement de la manne soviétique, certains services sociaux et sanitaires par le biais d'organismes tel Caritas (Quigley T., 2003 : 99-100).

Le fait que Fidel proclame en 1992 la fin du caractère athée du régime et qu'il se rende à Rome en 1996 ouvre la voie au voyage de Jean-Paul II de 1998, provoquant l'aire de l'administration Clinton, qui, comme ses prédécesseurs, continue d'appliquer une stricte politique d'isolement de Cuba et d'encouragement à la mobilisation de la société civile, et ce malgré l'effondrement du principal bailleur de fonds des Castro, à savoir l'Union soviétique, en 1991 (Capote S., 2012).

15 L'approche employée par le pape lors de son voyage n'est pas celle d'une remise en cause du régime. Si Jean-Paul II critique l'idéologie marxiste qui le sous-tend et enjoint Cuba à s'" ouvrir au monde ", le fait que le pape apparaisse aux côtés du Líder Máximo et montre du doigt, parmi les causes de «la pauvreté matérielle et morale [qui frappe le pays]», "les mesures économiques restrictives injustes et éthiquement inacceptables imposées par l'étranger " ${ }^{3}$, indique qu'il ne souhaite pas adopter une stratégie d'affrontement (Jean-Paul II, 1998). Le but du voyage est avant tout d'accroître la liberté religieuse sur l'île et dans ce contexte, il est préférable d'opter pour une attitude plutôt conciliante.

Dès lors, l'approche du Saint-Siège consiste à renforcer la présence de l'Église grâce à un dialogue avec les autorités, ce qui exclut de fait qu'elle se pose en pourfendeur du gouvernement cubain. Le Saint-Siège, loin de soutenir publiquement les initiatives des dissidents, se montre même assez méfiant, surtout quand ces derniers sont catholiques, car comme l'a expliqué un diplomate romain à ses homologues américains, le Vatican ne veut surtout pas que l'Église catholique soit perçue comme faisant partie de l'opposition. Il attribue d'ailleurs la lenteur des progrès en matière de liberté ecclésiale à l'action de dissidents revendiquant haut et fort leur foi catholique ${ }^{4}$. La question des droits humains, et surtout le sort des prisonniers politiques, est bien abordée, mais en privé (Gaetan V., 2012). L'approche s'avère plutôt payante pour le catholicisme sur l'île (Alonso, A., 2009: 295), qui connaît un rebond des vocations, un séminaire ayant même pu ouvrir ses portes en novembre 2010 en présence de Raul Castro (Ortega J., 2012). L'Église est devenue un acteur clé de la société cubaine, s'acquittant de missions sociales que l'État peine à assurer ${ }^{5}$. De plus, outre une certaine "renaissance » de l'Église, l'approche suivie par Jean-Paul II et son successeur a eu pour effet d'en faire la seule interlocutrice indépendante du régime, dans un pays où l'opposition est faible et fragmentée (Contreras-Garcia D., 2012: 177). Le mouvement catholique laïc connaît 
également une progression sans précédent, marquée par la multiplication d'organisations et de publications très dynamiques (Alonso A., 2009 : 295).

\section{L'opportunisme vain des États-Unis face au rapprochement entre le Saint-Siège et La Havane}

Les évolutions dans les relations entre le Vatican et Cuba n'échappent pas aux autorités américaines qui, après avoir redouté les conséquences d'un rapprochement entre le Saint-Siège et La Havane, commencent à penser au Vatican comme catalyseur d'un possible changement politique sur l'île ${ }^{6}$. L'approche américaine vis-à-vis de Cuba consiste toujours à isoler La Havane tout en cherchant à dynamiser la société civile. Les dirigeants américains se souviennent du rôle joué par l'Église polonaise et le Vatican de Jean-Paul II dans les années 1980 ; c'est dans cet esprit qu'ils espèrent s'adjoindre l'aide $\mathrm{du}$ Vatican dans ces tentatives vis-à-vis de Cuba, et ce alors que la position et l'influence de l'Église à Cuba et en Pologne soient très différentes (Crahan M., 2003 : 1-4). L'administration de George W. Bush, la première à espérer tirer profit des retombées du voyage papal, demande au Saint-Siège non seulement d'intervenir pour faire libérer les dissidents, mais aussi d'exhorter La Havane à rétablir les libertés fondamentales et à accepter des élections libres ${ }^{7}$. Ces requêtes voient leur fréquence et leur ambition s'intensifier après le passage de flambeau de Fidel Castro à son frère, Raul, en 2006, car les États-Unis espèrent que cette transition puisse être l'occasion d'un changement plus radical ${ }^{8}$.

Le Saint-Siège laisse ces requêtes sans réponse, au grand dam de ses interlocuteurs américains, dont ce conseiller politique de l'ambassade près le Saint-Siège qui, en 2002, s'étonne auprès de ses homologues romains que l'Église ne joue pas un rôle aussi actif pour la liberté qu'elle ne l'a fait en Pologne du temps de Jean-Paul $\mathrm{II}^{9}$. Or, "si la démocratie est actuellement la jauge unique des relations internationales dans le monde occidental », au point de justifier des interventions militaires, le Vatican, pour sa part, n'a "aucun projet politique messianique» pour reprendre les termes de l'historien Jean-Baptiste Noé. Il « ne cherche pas à faire advenir un régime par rapport à un autre » et a en ce sens une géopolitique « éminemment réaliste ». Là où nombre d'États cherchent à imposer leur idéal politique, le Saint-Siège favorise une politique des petits pas (expression d'ailleurs reprise par le pape François pour expliquer sa vision de la diplomatie) et de l'entente, « capable de transiger sur le superflu pour rester ferme sur le nécessaire » (Noé J.-B., 2015 : 224-225). Ce réalisme le rend capable de dialoguer avec des pays aux visions du monde différentes de la sienne. Comme l'ont pu le constater les diplomates américains, bien que « d'un point de vue philosophique favorable à une transition démocratique sur l'île et à une plus grande liberté pour ses habitants ", le Saint-Siège est soucieux avant tout de ménager un espace d'exercice pour sa mission, espace qu'il risque de perdre s'il se met à dos le gouvernement cubain par un activisme politique trop audacieux aux yeux de ce dernier. Les diplomates américains retiennent de leur entretien avec leurs homologues catholiques les termes de "patience » et de "prudence » dans l'approche vis-à-vis de Cuba. Ainsi, alors que la diplomatie américaine demande au Saint-Siège de protester lors des arrestations massives de 2003, le diplomate en charge de Cuba au Saint-Siège répond que l'Église se trouve dans une "situation délicate" du fait de la nécessité de protéger sa fragile présence sur l'île ${ }^{10}$. Les diplomates du Vatican expliquent en 2002 aux Américains qu'ils pensent que le changement politique sur l'île sera lent. S'ils jugent une transition 
« inévitable ", ils estiment qu'elle pourrait ne se produire que dans de nombreuses années, y compris "des années après que Fidel Castro eut quitté la scène ${ }^{11}$. Contrairement à Washington, Rome semble même dans une certaine mesure redouter une transition démocratique dans l'île, surtout si celle-ci devait se produire dans un avenir proche. Comme l'explique l'un des responsables de la diplomatie du Saint-Siège Mgr Pietro Parolin en 2006 à ses homologues états-uniens, la liberté politique n'entraîne pas nécessairement une plus grande piété ou une plus grande implication dans l'Église. Le Vatican craint que dans une île comme Cuba, où l'Église n'a jamais été très enracinée, une transition rapide vers la démocratie ne lui soit fatale ${ }^{12}$. Il préfère une transition en douceur, sur le modèle de l'Espagne à la mort de Franco. Jean-Paul II était d'ailleurs profondément déçu des conséquences de la chute du rideau de fer chez ses concitoyens polonais, qui s'empressèrent selon lui d'adhérer aux valeurs matérialistes occidentales ${ }^{13}$. Pour Parolin, la «nouvelle ère de liberté constituerait un défi pour l'Église $»^{14}$, qui craint aussi de payer le prix de sa politique d'accommodement avec les Castro une fois que ces derniers auront quitté la scène (Gaetan V., 2012).

\section{L'administration Obama, ou l'occasion d'un reset entre Washington et La Havane?}

\section{Au Vatican, les espoirs déçus du premier mandat Obama}

19 L'arrivée de Barack Obama à la Maison-Blanche en janvier 2009 marque un changement dans la politique poursuivie par les États-Unis vis-à-vis de Cuba. Dès la campagne pour l'élection présidentielle, le candidat démocrate avait appelé de ses vœux une nouvelle approche, qualifiant d'échec la politique poursuivie depuis cinquante ans par Washington. En lieu et place de la politique d'isolement du régime et d'encouragement de la société civile pratiquée par ses prédécesseurs, le président Obama déclare lors du sommet des Amériques d'avril 2009 vouloir prendre un nouveau départ dans ses relations avec le régime et assortit ses déclarations de signes concrets de détente. Raul Castro pour sa part se déclare prêt au dialogue, mais sur « un pied d'égalité et dans le respect absolu de la souveraineté cubaine et de son droit à l'autodétermination » (LeoGrande W., 2011: 38). L'approche progressive de l'administration Obama dans le rétablissement des liens avec Cuba est initialement bien accueillie par Raul Castro.

Le Vatican se félicite de cette nouvelle approche, bien plus en phase avec celle qu'elle poursuit depuis le voyage de Jean-Paul II $^{15}$. Depuis 1998 d'ailleurs, les diplomates catholiques n'ont eu de cesse d'exhorter leurs homologues états-uniens à changer leur politique vis-à-vis de Cuba, à " dialoguer » avec le gouvernement au lieu de l'isoler avec un embargo inefficace, qu'ils jugent "anachronique $»^{16}$. La levée de toute sanction, font-ils valoir, serait non seulement une mesure «juste", mais aussi «intelligente", car elle priverait le gouvernement cubain d'excuse pour ses mauvaises performances économiques, ce qui pourrait conduire à des réformes politiques ${ }^{17}$. L'accession au pouvoir de Raul Castro avait d'ailleurs conduit le Vatican à redoubler d'appels au gouvernement américain à dialoguer avec son homologue cubain. En 2008, c'est le secrétaire d'État, le cardinal Tarcisio Bertone, qui avait confié à l'ambassadrice américaine près le Saint-Siège, Mary Ann Glendon, que « Raul est un homme à l'esprit pratique disposé à prendre des mesures pratiques $»^{18}$, tandis que le diplomate Angelo 
Accattino insistait auprès de ses interlocuteurs américains que «l'heure du dialogue était venue » et les enjoignait à se garder de tout propos polémique ${ }^{19}$.

Le Vatican espère également qu'un rapprochement entre Washington et La Havane permettra de fissurer le front qui est en train de se constituer entre les régimes de gauche d'Amérique latine. Alors que de grands progrès ont été effectués à Cuba, le Saint-Siège redoute qu'outre le Venezuela, où les tensions avec l'Église sont vives, d'autres pays de la région soient touchés par une révolution de type bolivarienne. Encouragé par le dégel voulu par les Américains, le Saint-Siège exhorte ces derniers à faire preuve d'encore plus d'audace, quand il leur suggère par exemple un geste de clémence envers les Cuban Five, ces hommes condamnés à de lourdes peines pour espionnage et détenus aux Etats-Unis (en autorisant l'entrée de leurs épouses sur le territoire américain), voire la prise en compte de la proposition de Castro de les échanger contre des prisonniers politiques ${ }^{20}$.

Le dégel entre Washington et La Havane n'est que de courte durée cependant. Malgré les signes positifs des débuts de la présidence Obama, les tensions reprennent vite le dessus. L'action concrète de Washington (le dossier cubain n'y est pas une priorité ; Cuba est maintenue sur la liste des États sponsors du terrorisme ; 20 millions de dollars sont encore alloués à l'agence américaine de développement USAID pour la "promotion de la démocratie " sur l'île) semble contredire la volonté initiale de dialogue manifestée par Barack Obama (LeoGrande W., 2011 : 39-40). L'arrestation en décembre 2009 du sous-traitant d'USAID, Alan Gross, accusé d'espionnage par Cuba courrouce les États-Unis, qui imposent de nouvelles mesures restrictives, parmi lesquelles l'ajout de Cuba à une liste de quatorze pays soumis à des contrôles aéroportuaires renforcés. Plus généralement, les Cubains s'indignent que la matrice de la politique américaine demeure la même : celle de favoriser à terme un changement de régime en faveur de la démocratie. Quoi qu'il en soit, la volonté américaine de promouvoir le regime change, même à un tempo réduit, rend tout dialogue réel impossible.

Le Vatican déplore ces tensions et s'impatiente. En janvier 2010, le père Accattino, citant en exemple la décision concernant les aéroports, indique aux diplomates américains en poste au Saint-Siège que les États-Unis devraient s'abstenir de toute mesure unilatérale contre Cuba car cela fait le jeu de Castro et de Chavez, qui s'en servent comme preuves de l'hostilité intrinsèque des États-Unis à l'égard de Cuba. Le Vatican craint que la situation catastrophique de l'économie cubaine ne débouche sur un bain de sang. Une fois de plus, le diplomate catholique rappelle que le réel danger dans la région n'est plus Cuba, mais Chavez, et qu'il faut absolument dialoguer avec Castro pour contrer la menace représentée par le Venezuela. Le Saint-Siège critique également la stratégie des deux poids deux mesures menée par les États-Unis, qui refusent tout dialogue avec Cuba tant qu'il n'y aura pas d'avancées en matière de droits humains, tout en dialoguant avec d'autres pays peu soucieux de ces questions, comme la Chine. Enfin, le diplomate du Vatican exhorte les Américains à ne pas se laisser prendre en otage par des considérations de politique intérieure, en l'occurrence par la pression du lobby cubano-américain, qu'il tient pour responsable de "politiques contreproductives ", comme celle de renforcer les contrôles aéroportuaires ${ }^{21}$.

Dans ce contexte, le voyage qu'effectue le pape Benoît XVI en mars 2012 à Cuba vient en tout cas rappeler que la position du Saint-Siège demeure inchangée. Les États-Unis ne sauraient compter sur l'Église pour des appels à la transition politique, la priorité du 
pape demeurant la survie de l'institution. Le voyage a d'ailleurs été prévu pour régler certains problèmes ecclésiaux, comme la baisse du niveau de pratique religieuse après un rebond initial (Donadio R. et R. Archibold, 2012) au profit des Églises évangéliques et pentecôtistes (Archibold R., 2012). Il s'agit aussi de renforcer le rôle de l'Église dans l'enseignement. Les propos du pape sont donc avant tout de nature pastorale. Si sa prière pour ceux qui sont " privés de liberté et séparés de leurs proches " au sanctuaire de la Vierge de la Charité du Cuivre constitue une référence claire aux prisonniers politiques, le pape ne manque pas de critiquer l'embargo américain lors de son discours d'adieu (Benoît XVI, 2012b) et lors de son homélie Place de la Révolution, il reconnait " avec joie " qu'à Cuba, "des mesures ont été prises pour permettre à l'Église » de s'acquitter de sa mission « ouvertement et publiquement » (Benoît XVI, 2012a). Il refuse de rencontrer une délégation des "Dames en Blanc», ces épouses de dissidents emprisonnés, alors que ces dernières avaient demandé une audience (Allen J., 2014b). La question du sort des dissidents, ainsi que celui d'Alan Gross, aurait bien été abordée avec Raul Castro, mais en privé (Wooden C., 2012 ; Boorstein M., 2014).

\section{L'avènement du pape François et le second mandat Obama : un concours de circonstances propice au changement}

25 Après sa réélection en 2012, Barack Obama souhaite repartir sur de bonnes bases et faire de l'amélioration des relations avec Cuba une des réussites de sa présidence, car il anticipe ou constate l'échec de la plupart de ses grands chantiers de politique étrangère, dont les reset avec la Russie et la Chine. Le début de son deuxième mandat coïncide, à quelques semaines près, avec l'élection d'un nouveau pape, François, en mars 2013, après la renonciation de Benoît XVI. La présence concomitante des deux hommes au pouvoir, ainsi que les circonstances à Cuba, entre crise économique et un certain pragmatisme de la part de Raul Castro, vont permettre une avancée sans précédent sur le dossier.

Si le Vatican a une longue histoire de bons offices à son actif, la personnalité du nouveau pape n'est pas étrangère à la décision du Saint-Siège de contribuer à sa manière au rapprochement entre Cuba et les États-Unis. Contrairement à son prédécesseur, François est féru de politique étrangère et il entend bien replacer le Saint-Siège au centre du jeu diplomatique (Gibson D., 2014). Le fait qu'il soit le premier pape des Amériques a également été déterminant dans sa décision de s'impliquer dans ce processus. L'importance que revêt le continent à ses yeux s'est manifestée par la nomination - ou reconduction - à des postes clés du Vatican de fins connaisseurs de la région. Pietro Parolin, son secrétaire d'État, diplomate de formation, a notamment été nonce au Venezuela entre 2009 et 2013. François confirme au poste de substitut pour les Affaires générales, numéro deux de la secrétairerie d'État, Giovanni Becciu, qui a été nonce à La Havane de 2009 à 2011. Le sous-secrétaire pour les relations avec les États, Mgr Antoine Camilleri, a quant à lui été nonce à Cuba en 2005-2006 (Pullella P., 2014).

Jorge Mario Bergoglio s'intéresse en outre depuis longtemps à Cuba à titre personnel. S'il n'a pu y accompagner le pape lors de son voyage de 1998 car il était retenu à Buenos Aires face à l'imminence du décès de l'archevêque auquel il était appelé à succéder, il n'en a pas moins écrit un ouvrage intitulé Dialogues entre Jean-Paul II et Fidel Castro dans lequel il critique avec force le système socialiste, mais également l'embargo américain, 
qui conduit à l'isolement de l'île et son appauvrissement. Il entretient depuis 1981 des relations étroites avec le primat de l'Église catholique à Cuba, le cardinal-archevêque de La Havane, Jaime Ortega, (Colonna-Cesari C., 2016).

De son côté, Raul Castro, qui a introduit depuis son arrivée au pouvoir en 2006 certaines réformes économiques, se déclare ouvert au dialogue, à condition que la nature du régime ne fasse pas l'objet des conversations. Ses motivations à dialoguer sont avant tout pragmatiques, puisque les progrès économiques qui auraient dû suivre ses réformes tardent à se concrétiser, et la manne du bienfaiteur vénézuélien se tarit avec la chute des cours du pétrole; les devises apportées par les touristes américains pourraient redonner un peu d'air à une économie en grande difficulté (The Economist, 2015).

\section{La médiation pontificale : circonstances exceptionnelles et continuité diplomatique}

\section{Étude d'une médiation pontificale}

Dans ce contexte favorable au dialogue, des négociations sont entamées dans le plus grand secret en juin 2013 entre les États-Unis et Cuba, portant notamment du côté américain sur la libération d'Alan Gross et du côté cubain sur celle des trois Cuban Five toujours en détention. L'équipe Obama espère également une avancée encore plus significative, telle que le rétablissement de relations diplomatiques, qui constituerait un élément de poids dans son héritage politique. Plusieurs parlementaires américains, qui ne sont pas au courant des négociations, jugent de leur côté que le moment est venu pour un réel changement de politique envers Cuba. C'est notamment le cas du président pro tempore du Sénat, le démocrate et catholique Patrick Leahy, qui souhaite favoriser l'intervention du pape François. A cette fin, le sénateur écrit en mars 2015 à plusieurs cardinaux réputés pour être proches de François. Il s'agit d'inciter ces derniers à demander au pape qu'il évoque entre autres points, la question des prisonniers lors de l'audience prévue avec le président Obama à Rome le 27 mars. Les lettres sont destinées tout d'abord au cardinal-archevêque émérite de Washington, Theodore McCarrick et au cardinal-archevêque de Boston, Sean O'Malley, qui connaît bien l'Amérique latine et Cuba, où il s'est rendu à de nombreuses reprises (Kornbluh $\mathrm{P}$. et $\mathrm{W}$. LeoGrande, 2015b) et elles sont bien accueillies par les deux prélats. Leur intervention auprès du pape s'inscrit dans une longue histoire d'implication de l'épiscopat des États-Unis dans les relations cubano-américaines, rôle qui a complété et alimenté celui joué par le Saint-Siège. Dès 1972, à la demande de leurs homologues cubains, les évêques américains demandaient la fin de l'embargo. Pendant les années 1980 et 1990, le diocèse de Boston joue déjà un rôle important dans ces efforts de réconciliation du fait de l'action du cardinal Bernard Law (Quigley T., 2003 : 98). Par ailleurs, les évêques états-uniens sont en partie responsables de l'amorce d'un dialogue entre le gouvernement et l'épiscopat cubains, car ils exigèrent au cours de leur premier déplacement sur l'île, en janvier 1985, la présence d'évêques cubains lors de toute entrevue avec Fidel Castro (Quigley T., 2003 : 97).

L'audience entre Barack Obama et François se déroule le 27 mars. Elle dure près d'une heure, le double du temps alloué, et porte essentiellement sur Cuba, sujet sur lequel le pape serait revenu à plusieurs reprises (Vallely P., 2015 : 392). Pressé par le pape qui lui 
dit qu'il était "grand temps de procéder à un changement", notamment sur la question de l'embargo (Parsons C. et M.A. Memoli, 2015), Obama aurait informé le pape que les États-Unis avaient " quelque chose en cours avec Cuba ", dont les négociations mises en place en juin 2013. Obama signifie à François qu'il serait utile qu'il joue un rôle dans ce dialogue (Kornbluh P. et W. LeoGrande, 2015a). Le pape a ensuite la surprise de voir le président acquiescer à ses critiques concernant le caractère contreproductif de l'embargo (Oppmann P., 2015), même si le président lui rappelle que sa levée relève du Congrès. Barack Obama ajoute qu'il existe des obstacles à l'amélioration des relations entre les deux pays, en l'occurrence la détention de Gross et des trois Cuban Five. Le pape avance qu'un "geste de clémence envers les Cubains détenus dans les prisons américaines serait certainement suivi d'un geste réciproque par le gouvernement cubain concernant le prisonnier américain qui y est détenu» (Ortega J., 2017a : 109). Mgr Ortega estime que le pape déduisit de cet entretien qu'il pourrait contribuer à aider à « surmonter les obstacles » évoqués par B. Obama.

31 Le sénateur du Vermont Patrick Leahy avait également fait parvenir une missive au cardinal-archevêque de La Havane, Jaime Ortega y Alamino, pour le prier instamment de demander au pape de soulever la question cubaine avec le président lors de cette même audience (Ortega J., 2017 : 98). C'est d'ailleurs sur le cardinal Ortega que le pape va porter son choix pour faire la navette entre les deux parties et les aider à "surmonter [l]es obstacles ». Cette décision n'est pas étonnante quand on connaît le parcours du prélat cubain. Il a plusieurs fois eu l'occasion de se rendre à Washington pour des visites à ses homologues de l'épiscopat états-unien. Très souvent, ces voyages furent l'occasion de nouer des contacts avec des représentants des autorités politiques des États-Unis. Ainsi, dès le mois de septembre 1985, il fut reçu aux côtés d'autres prélats cubains au département d'État et à la Maison-Blanche. En 2010, il joue un rôle clé dans la libération de plusieurs prisonniers politiques de 2003, rôle qui le conduit une fois de plus à Washington où il est reçu par le conseiller à la sécurité nationale, James Jones, et le secrétaire d'État adjoint en charge de l'Amérique latine, Arturo Valenzuela (Espacio laical, 2010 : 132). Côté cubain, Mgr Ortega devient en 1994 le premier cardinal créé sur l'île depuis plusieurs dizaines d'années, mesure prise pour redynamiser l'Église, mais également pour donner au régime un interlocuteur de haut niveau (Alonso A., 2009 : 297). En outre, comme nous l'avons vu supra, Mgr Ortega et Jorge Mario Bergoglio se connaissent depuis 1981 et les deux ecclésiastiques sont proches.

32 Tout ceci permet de comprendre pourquoi François demande à voir le cardinal cubain alors que ce dernier est à Rome pour assister à la canonisation de Jean XXIII et JeanPaul II fin avril 2013. Mgr Ortega apprend à cette occasion par la voix du secrétaire d'État Mgr Parolin que les autorités cubaines ont demandé au pape d'intervenir sur la question de l'échange de prisonniers (Ortega J., 2017 : 104). Lors de leur entrevue, début mai, Mgr Ortega et François évoquent les obstacles à l'avancée des négociations, notamment le refus des États-Unis d'échanger un seul Américain contre trois Cubains, d'autant qu'ils nient que Gross ait été un espion ${ }^{22}$. Les deux ecclésiastiques concluent que face au blocage des négociations dû aux "obstacles », il est urgent pour le Vatican d'agir, d'autant que la fin du second mandat d'Obama approche à grands pas. Il ne s'agit pas pour le pape d'intervenir directement et concrètement dans les démarches de libération. Il accepte cependant la suggestion faite par le sénateur Leahy d'écrire aux présidents Obama et Castro (Ortega J., 2017a : 115) et charge le cardinal Ortega de remettre ces lettres en mains propres à leurs destinataires respectifs afin que le prélat 
puisse leur fournir des précisions quant à la pensée du pape. Par exemple, le pontife donne pour instruction au cardinal de suggérer à Raul Castro, quand il lui remettra la lettre, de faire le premier pas en libérant Alan Gross, car les États-Unis agiraient sans doute dans la foulée de manière réciproque. Interrogé plus tard sur les termes exacts de la lettre de François, dont l'intégralité du contenu n'a toujours pas été rendue publique, Jaime Ortega a d'ailleurs répondu que « la lettre, c'était moi ». Elle aurait en effet été de nature très générale, et les points précis auraient été abordés oralement par le cardinal (Ortega J., 2017c). Tout au plus sait-on qu'elle apportait le soutien du pape aux négociations en cours, et encourageait les deux dirigeants à résoudre "les questions humanitaires d'intérêt commun, y compris la situation de certains prisonniers, afin d'entamer une nouvelle phase dans leurs relations " (Kornbluh P. et W. LeoGrande, $2015 \mathrm{~b}: 444-445$ ). En outre, le pontife y proposait les services du Vatican (Kornbluh P. et W. LeoGrande, 2015a).

De retour à Cuba, Mgr Ortega réfléchit aux modalités de remise des lettres, qui s'avèrent plus délicates pour Obama que pour Castro ${ }^{23}$. L'administration Obama pour sa part souhaite que l'opération se fasse dans la plus grande discrétion pour ne pas susciter de rumeurs quant à un éventuel dialogue avec Cuba. La présence du cardinal Ortega à la Maison-Blanche est ainsi jugée inopportune (Ortega J., 2017a : 121-122). Face à l'insistance du prélat cubain, une rencontre est organisée entre ce dernier et Barack Obama à la Maison-Blanche le 17 août 2013, le cardinal McCarrick ayant organisé un séminaire à l'université de Georgetown avec pour invité Mgr Ortega, afin de justifier la présence de ce dernier dans la capitale (Ortega J., 2017a: 125; Kornbluh P. et W. LeoGrande, 2015a). Quelques jours avant son déplacement à Washington, et conformément aux instructions de François, le cardinal cubain se rend sur le lieu de villégiature de Raul Castro pour lui remettre le pli pontifical, que le dirigeant cubain aurait accueilli très favorablement, avant de demander à Mgr Ortega de profiter de son déplacement à Washington pour signaler au président Obama que Cuba était prête à prendre des mesures pour améliorer les relations avec les États-Unis (Hirayama A., 2017). La décision de Raul Castro de demander à Mgr Ortega de faire passer un message au président des États-Unis montre bien l'intérêt d'une médiation portée par un prélat bénéficiant de la confiance et de l'estime de chacune des parties, ainsi que l'importance des rencontres en face à face pour régler les points les plus épineux qu'une simple lettre ne saurait résoudre.

Mgr Ortega, porteur de la lettre du pape et du message oral du président cubain, se rend ensuite à la Maison-Blanche comme convenu. Là encore, l'importance que revêt la discrétion aux yeux de la Maison-Blanche dans le dialogue avec Cuba se manifeste par le fait que le nom du cardinal ait été délibérément omis des registres de visiteurs à la Maison-Blanche (Hirayama A., 2017). Mgr Ortega transmet à Barack Obama le message de Raul Castro, dont se félicite le président, ainsi que la lettre de François. Obama juge «très utile » que le pape s'intéresse à la question (Ortega J., 2017 : 127-128). Le cardinal cubain évoque ensuite les suggestions plus concrètes émises par le pape lors de leur entrevue de mai, François et Mgr Ortega jouant là-encore chacun leur partition : lettre à caractère général de la part du pape et solutions pratiques de la part de son représentant. Le médiateur est aussi chargé par B. Obama de transmettre à R. Castro un message d'optimisme et d'espoir quant à l'amélioration des relations entre leurs deux pays d'ici la fin de sa mandature (Ortega J., 2017c). 

pape dans sa lettre en choisissant le Vatican comme lieu de signature de l'accord final entre les deux États, qui a lieu le 28 octobre 2013 en présence de quatre hauts responsables catholiques, dont le secrétaire d'État, Mgr Parolin et le cardinal Ortega (Kornbluh P. et W. LeoGrande, 2015b : 445-446). Chaque délégation a tout d'abord une brève rencontre seule à seule avec les dignitaires catholiques, puis elles annoncent à leurs hôtes romains, à la grande surprise de ces derniers, qu'outre la libération des prisonniers, les deux pays ont décidé du rétablissement de relations diplomatiques (Landler M., 2016).

De l'avis de ses participants, il ne s'agissait pas avec cette rencontre de résoudre des questions restées en suspens avant de parvenir à un accord. Le fait qu'elle se déroule au Vatican avait surtout pour but d'instaurer la confiance entre les deux parties par la présence de ce médiateur au statut si particulier qu'est le Saint-Siège (Kornbluh P. et W. LeoGrande, 2015a). En outre selon Mgrs Ortega et Becciu, par leur présence, les représentants du pape assumaient ainsi un rôle de garants du respect de l'accord (TV2000, 2015; Hirayama A., 2017). Le fait que le 17 décembre, anniversaire de François, ait été choisi pour annoncer le rapprochement, aurait d'ailleurs été, de l'avis de ces mêmes prélats, un moyen de rendre hommage au rôle joué par le pontife pendant ce processus (Ortega J., $2017: 10$ ).

Outre leur contribution à un déblocage des négociations, les bons offices du pape auraient également été recherchés, à Washington et à La Havane, pour ce qu'ils offraient de "protection symbolique » aux deux dirigeants dans leurs pays respectifs. Ainsi, la popularité de François aux États-Unis aurait en elle-même constitué une assurance pour Obama vis-à-vis de ses détracteurs, parmi lesquels ceux qui pourraient s'insurger contre un rapprochement avec des « communistes athées ", notamment dans le camp républicain. Du côté cubain, le fait que le pape soit latino-américain permettait aussi de protéger d'une certaine manière Raul Castro au moment de signer un accord avec les "capitalistes américains"; les négociateurs cubains auraient confié sans équivoque à leur homologue américain Benjamin Rhodes qu'ils considéraient François, le premier pape des Amériques, comme différent de ses prédécesseurs (Dias E., 2015 : 28 ; Vallely P., 2015 : 392).

Le pape a cherché à minimiser son action. En juillet 2015, à bord du vol qui le ramène du Paraguay, il nie qu'il y ait eu médiation de sa part et attribue tout le mérite de l'issue du dialogue à ses deux protagonistes, déclarant que « nous n'avons rien fait » (Ortega J., 2017 : 154). Il reconnaît cependant à demi-mots son intervention, qu'il impute à l'inspiration divine : "Que pouvais-je faire avec ces deux pays qui se comportaient de la sorte depuis 50 ans? ", a-t-il répondu aux journalistes. "Puis, le Seigneur m'a fait penser à un cardinal ", faisant allusion à Mgr Ortega et au rôle de médiateur qu'il lui a confié. Il ajoute qu'ils n'ont " pas fait grand chose, seulement de petites choses " (Dias E., 2015 : 28). Cette intervention s'inscrit donc dans la "diplomatie des petits pas ", comme François aime à qualifier son approche. De plus, elle participe pleinement de sa conception de l'action diplomatique telle qu'il l'avait présentée lors de son premier discours au corps diplomatique, en janvier 2014, conception qui repose sur « la voie diplomatique du dialogue » (Pape François, 2014). 


\section{Une médiation qui s'inscrit dans une longue tradition diplomatique}

39

intervention, en dépit des dénégations du pape François quant à l'ampleur de son action, a pour effet de replacer le Saint-Siège au premier plan de la scène diplomatique. Mais si sur la forme elle constitue une rupture par rapport à la position de retrait adoptée par Jean-Paul II à la fin de son pontificat, et surtout avec celle de Benoît XVI, on ne saurait parler de rupture radicale dans l'approche du Vatican sur le fond, notamment pour ce qui est des motivations et des objectifs de son action diplomatique. Pour exceptionnelle qu'ait été cette médiation, fruit d'un concours de circonstances inédit à Washington (avec un président soucieux de son héritage politique), à La Havane (avec un président plus pragmatique et dont l'économie était aux abois) et au Vatican (avec le premier pape des Amériques), elle ne doit pas occulter le fait qu'elle s'inscrit dans la longue tradition de bons offices dans la région, mais surtout qu'elle reste de par ses objectifs conforme aux principes qui régissent la diplomatie vaticane depuis la fin du XIXe siècle : promotion de la paix et du dialogue, certes, mais aussi et peut-être avant tout défense des intérêts de l'institution ecclésiale et des fidèles quitte à accepter certains compromis - et refus de tout «messianisme politique » comme l'a indiqué Jean-Baptiste Noé cité supra.

La diplomatie du nouveau pontife a souvent été présentée comme au diapason de celle de Barack Obama, notamment du fait de leur entente sur certains sujets comme la lutte contre le réchauffement climatique. Le fait que François ait proposé ses bons offices, après que le Saint-Siège eut longtemps refusé toute intervention à caractère politique au sujet de Cuba, ne signifie en rien un alignement de la diplomatie du Saint-Siège sur celle de la Maison-Blanche. Bien au contraire, la continuité des positions du Vatican sur certains dossiers depuis l'élection de François a donné lieu à certains désaccords entre les deux hommes, notamment sur la question du Moyen-Orient, où la position de l'Église demeure largement conditionnée par le souci de protéger les minorités chrétiennes et de garantir leur présence pérenne ; elle est d'ailleurs influencée par l'avis de ces mêmes minorités, pour lesquelles un dirigeant fort, voire issu des forces armées, constitue le meilleur rempart contre le radicalisme islamiste. On a donc pu voir François condamner d'éventuelles frappes américaines sur la Syrie après le franchissement de la tristement célèbre "ligne rouge » à l'été 2013 et constater ses réserves concernant un changement de régime à Damas, le pape craignant que son départ entraîne le chaos (Allen J., 2014a). Cette posture rappelle l'opposition virulente du pape Jean-Paul II au déclenchement des deux guerres en Irak. Cette inquiétude pour les chrétiens d'Orient a également conduit François à écrire à Vladimir Poutine à l'occasion du sommet du G20 de Saint-Pétersbourg en septembre 2013 pour lui demander de tout faire pour que les dirigeants mondiaux renoncent à l' "option militaire » contre la Syrie. Son souci de protéger l'Église est encore à l'œuvre quand il se fait remarquer pour sa "discrétion » lors des interventions russes en Syrie et en Ukraine - et ce en dépit d'un lobbying américain intensif - discrétion qui serait motivée par la volonté de ne pas fragiliser les relations avec le patriarche de l'Église orthodoxe avec lequel le Vatican cherche un rapprochement depuis très longtemps, Cette stratégie semble avoir été couronnée de succès, puisque les deux hommes se sont rencontrés enfin en 2016 - à Cuba - après des siècles de vives tensions (Reese T., 2017). 


\section{Conclusion}

Le voyage du pape François à Cuba en septembre 2015 a apporté une preuve supplémentaire que le premier pontife d'Amérique latine restait préoccupé, comme ses prédécesseurs, du sort de l'église dans l'île et qu'il ne cherchait pas nécessairement par son intervention à précipiter un changement de régime à La Havane. En amont de ce voyage, l'archevêque de Miami, Thomas Wenski, confiait d'ailleurs qu'à Cuba l'« Église souhaitait un atterrissage en douceur, car l'alternative serait le chaos » (San Martin I., 2015), ce qui n'est pas sans rappeler les propos des diplomates catholiques au cours de leurs entretiens avec leurs homologues américains du temps du pontificat de Benoît XVI. Lors de ses nombreuses prises de parole à Cuba, le pape François s'est efforcé de trouver un difficile équilibre entre la liberté pour l'Église d'exercer sa mission et la nécessaire défense des libertés politiques, s'attirant ainsi les foudres des CubainsAméricains. Il ne rencontra pas de dissidents et ne formula que des critiques modérées des violations des droits humains perpétrées par le régime, tout en n'oubliant pas de renouveler la critique de l'embargo américain.

Interrogé par Mgr Ortega au sujet de la situation des tensions croissantes entre l'Église et certains gouvernements latino-américains le matin même de son élection au siège de Pierre en mars 2013, François avait répondu que «face à ces nouveaux projets économiques et sociaux et les changements qu'ils apportent, l'Église ne peut rester simple spectatrice ; elle ne peut pas pour autant affronter la situation de l'extérieur par des critiques excessives ». Ces évolutions économiques et politiques doivent selon lui être accompagnées depuis l'intérieur par le biais du dialogue (Ortega J., 2017 : 48-49). Pour Mgr Ortega, ces propos rappellent fortement ceux que lui avait tenus en juin 2012 Benoît XVI, pour lequel «la seule voie, c'est le dialogue; l'Église n'est pas dans le monde pour changer les gouvernements, mais pour pénétrer le cœur des hommes avec l'Évangile » (Ortega J., 2017 : 50). Cette insistance sur le rôle du dialogue comme outil de changement, plutôt que sur l'affrontement, est centrale au pontificat de François. Pour Ortega, le pape François, loin d'être «une fleur exotique dans un jardin conventionnel " est l'héritier fidèle de Jean-Paul II et de Benoît XVI, eux-mêmes s'inscrivant dans la droite ligne de la diplomatie vaticane telle qu'elle est menée depuis la fin du XIXe siècle (Ortega J., 2017 : 53-54).

\section{BIBLIOGRAPHIE}

Allen, John (a), « When pope meets president, a 'reset' may not be in the cards », Crux, 2 mars 2014, https://cruxnow.com/church/2014/03/26/when-pope-meets-president-a-reset-may-notbe-in-the-cards/, page consultée le 25 novembre 2017.

Allen, John (b), « A victory for the Vatican's line of detente », Crux, 17 décembre 2014, https:// cruxnow.com/church/2014/12/17/us-cuba-breakthrough-is-a-victory-for-the-vaticans-line-ofdetente/?s_campaign=crux:email:daily, page consultée le 25 novembre 2017. 
Alonso Tejada, Aurelio, Iglesia y politica en Cuba revolucionaria, La Havane, Ed. Caminos, 2002. Alonso Tejada, Aurelio, El laberinto cubano tras la caida del muro, Buenos Aires, Clacso, 2009 ( $2^{\mathrm{e}}$ édition).

Archibold, Randal, « Catholic Church Deals with a Diminished Role in Cuban Life », New York Times, 25 mars 2012, http://www.nytimes.com/2012/03/26/world/americas/church-deals-witha-diminished-role-in-cuban-life.html, page consultée le 25 novembre 2017.

Barberini, Giovanni, Le Saint-Siège, sujet souverain de droit international, Paris, Cerf, 2003.

Benoît XVI (a), Homélie du pape, La Havane, 28 mars 2012, http://w2.vatican.va/content/ benedict-xvi/fr/homilies/2012/documents/hf_ben-xvi_hom_20120328_la-habana.html, page consultée le 25 novembre 2017.

Benoît XVI (b), Discours du pape, aéroport de La Havane, 28 mars 2012, http://w2.vatican.va/ content/benedict-xvi/fr/speeches/2012/march/documents/hf_ben-xvi_spe_20120328_congedocuba.html, page consultée le 25 novembre 2017.

Boorstein, Michelle, « Pope Francis Takes a Public Role in US-Cuban Relations », Washington Post, 17 décembre 2014, https://www.washingtonpost.com/local/pope-francis-takes-a-public-role-inus-cuba-relations/2014/12/17/9a394168-862f-11e4-a702-fa31ff4ae98e_story.html?utm_term=. 290bc4b5f31c, page consultée le 25 novembre 2017.

Capote, Salvador, « alvador, "nt-Siège, Mgr Becciu,es y el dialogo"s, 2015.ricaines?n cinq parties dressée par le entretiens avec leurs homologues Cuba y las relaciones Vaticano-Estados Unidos », America Latina in Movimiento, 13 avril 2012, http://www.alainet.org/es/active/54017, page consultée le 25 novembre 2017.

Cardinale, Igino, Le Saint-Siège et la diplomatie : aperçu historique, juridique et pratique de la diplomatie pontificale, Paris, Desclée de Brouwer, 1962.

Casaroli, Agostino, Il martirio della pazienza. La Santa Sede e i paesi comunisti (1963-1989), Turin, Einaudi, 2000

Castro, Maelis, «El Vaticano acepta mediar entre el chavismo y la oposición », El Pais, 14 septembre 2016, https://elpais.com/internacional/2016/09/14/america/ 1473874705_741250.html, page consultée le 25 novembre 2017.

Colonna-Cesari, Constance, Dans les secrets de la diplomatie vaticane, Paris, Seuil, 2016.

Contreras-Garcia, Delia, «Iglesia catolica y estado en la república de Cuba : pasado y presente de sus relaciones », America Latina Hoy, vol. 63, 2013, p. 177-195.

Crahan, Margaret, " Whither Cuba ? The Role of Religion », Woodrow Wilson Center Update on the Americas, $\mathrm{n}^{\circ} 14$, octobre 2003.

Day, Mark, « The Checkered History of Vatican Envoys », National Catholic Reporter, 2 October 1981 , p. 20.

Dias, Elizabeth, « The New Roman Empire », Time, 28 septembre 2015, p. 26-31.

Di Nolfo, Ennio, « L'Italie et la crise de Cuba en 1962 », in Maurice Vaïsse (dir.), L'Europe et la crise de Cuba, Paris, Colin, 1993, p. 109-122.

Donadio, Rachel et Randal Archibold, « Pope Calls for Authentic Freedom in Cuba », New York Times, 28 mars 2012, http://www.nytimes.com/2012/03/29/world/americas/pope-benedict-callsfor-authentic-freedom-in-cuba.html, page consultée le 25 novembre 2017.

Dupuy, André, La diplomatie du Saint-Siège, Paris, Téqui, 1980. 
Economist, The, « Why the United States and Cuba are Cosying Up », 29 mai 2015, https:// www.economist.com/blogs/economist-explains/2015/05/economist-explains-37, page consultée le 25 novembre 2017.

Espacio laical, «Cronología de la mediación del Cardenal Jaime Ortega Alamino, arzobispo de La Havana (tercera parte) », vol. 4, 2010, p. 132-134.

Falasca, Stefania, « Intervista. Parolin. Medio Oriente, la soluzione c'è, ma in realtà nessuno la vuole », Avvenire, 3 août 2017, https://www.avvenire.it/mondo/pagine/medio-oriente-lasoluzione-c-ma-in-realt-nessuno-la-vuole, page consultée le 25 novembre 2017.

Gaetan, Victor, «How the Catholic Church is Preparing for a Post-Castro Cuba », Foreign Affairs, 27 février 2012, https://www.foreignaffairs.com/articles/americas/2012-02-27/how-catholicchurch-preparing-post-castro-cuba, page consultée le 25 novembre 2017.

Gibson, David, « How Pope Francis's Diplomacy May Change Everything, Not Just US-Cuba Relations », Religion News Services, 18 décembre 2014, http://religionnews.com/2014/12/18/popefrancis-diplomacy-may-change-everything-not-just-us-cuba-relations/, page consultée le 25 novembre 2017.

Hirayama, Ari, Entretien avec le cardinal Jaime Ortega, « Cardinal tells inside story of landmark U.S.-Cuba deal », Asahi, 10 mai 2017, http://www.asahi.com/ajw/articles/AJ201705100014.html, page consultée le 25 novembre 2017.

Holbrook, Joseph, « The Catholic Church in Cuba, 1959-1962 : the Clash of Ideologies », International Journal of Cuban Studies, vol. 2, n³-4, automne-hiver 2010, p. 264-275.

Hurtado, Rogelio Fabio et Ted Henken, « Religion and Thought », in Ted Henken, Miriam Celaya et Dimas Castellanos (dir.), Latin America in Focus : Cuba, Santa Barbara, CA, ABC-Clio, 2013, p. 183-200.

Infobae, « El papa Francisco ofreció una mediación del Vaticano en Venezuela bajo 'unas condiciones muy claras' ", Infobae, 29 avril 2017, https://www.infobae.com/america/venezuela/ 2017/04/29/el-papa-francisco-ofrecio-una-mediacion-del-vaticano-en-venezuela-bajo-unascondiciones-muy-claras/, page consultée le 25 novembre 2017.

Jean-Paul II, Discours du Saint-Père Jean-Paul II, aéroport de La Havane, 25 janvier 1998, https:// w2.vatican.va/content/john-paul-ii/fr/speeches/1998/january/documents/hf_jp-

ii_spe_19980125_lahavana-departure.html, page consultée le 25 novembre 2017.

Kornbluh, Peter et William LeoGrande (a), « Fidel Castro has died. Here's an inside look at Cuba's crazy backchannel negotiations with Obama », Mother Jones, septembre-octobre 2015, http:// www.motherjones.com/politics/2015/08/secret-negotiations-gross-hernandez-kerry-popeobama-castro-cuba/, page consultée le 25 novembre 2017.

Kornbluh, Peter et William LeoGrande (b), Back Channel to Cuba : The Hidden History of Negotiations between Washington and Havana, Chapel Hill, The University of North Carolina Press, 2015.

Landler, Mark, Alter Egos: Obama's Legacy, Hillary's Promise and the Struggle over American Power, New York, Penguin, 2016.

Lecomte, Bernard, Le pape qui fit chuter Lénine, Tours, CLD Editions, 2007.

LeoGrande, William, « Making up is hard to do: Obama's 'New Approach' to Cuba ", Nacla Report on the Americas, juillet-août, vol. 44, nº4, p. 38-44. 
Létrillart, Philippe, «L'Eglise catholique et la 'société civile' à Cuba », Les études du CERI, n¹13, mars 2005, http://www.sciencespo.fr/ceri/sites/sciencespo.fr.ceri/files/etude113.pdf, page consultée le 25 novembre 2017.

Luxmoore, Jonathan et Jolanta Babuch, The Vatican and the Red Flag, Londres, Geoffrey Chapman, 1999.

McElwee, Joshua, « Obama thanks Pope Francis for help in US-Cuba deal”, National Catholic Reporter, 17 décembre 2014, https://www.ncronline.org/blogs/ncr-today/obama-thanks-popefrancis-help-us-cuba-deal, page consultée le 25 novembre 2017.

Melady, Thomas, The Ambassador's Story: The United States and the Vatican in World Affairs, Huntington, IN : Our Sunday Visitor Publishing Division, 1994, p. 79.

Noé, Jean-Baptiste, Géopolitique du Vatican, Paris, PUF, 2015

Obama, Barack, « Statement by the president on Cuba policy changes, 17 décembre 2014, https:// obamawhitehouse.archives.gov/the-press-office/2014/12/17/statement-president-cuba-policychanges, page consultée le 25 novembre 2017.

Oppmann, Patrick, « Pope Francis Heads to Cuba after Months of Secret Diplomacy », CNN,18 septembre 2015, http://edition.cnn.com/2015/09/18/world/pope-francis-cuba-diplomacy/ index.html, page consultée le 25 novembre 2017.

Ordaz, Pablo, « El papa ofrece mediar en el proceso de paz en Colombia », El Pais, 16 juin 2015, https://elpais.com/internacional/2015/06/15/actualidad/1434396571_992337.html, page consultée le 25 novembre 2017.

Ortega, Jaime, «La visita di un papa conciliatore e i suoi frutti », 30 giorni, 3 avril 2012, http:// www.30giorni.it/articoli_id_78335_l1.htm, page consultée le 25 novembre 2017.

Ortega, Jaime (a), Encuentro, Diálogo y Acuerdo, El papa Francisco, Cuba y Estados Unidos, Madrid, San Pablo, 2017

Ortega, Jaime (b), « Palabras pronunciadas por S.E.R. Cardenal Jaime Ortega Alamino, Arzobispo emérito de La Habana, en la presentación del libro "Encuentro, Diálogo y Acuerdo ", 16 juin 2017, http://www.arquidiocesisdelahabana.org/contens/noticias/ Discurso\%2016\%20de\%20junio\%202017\%20presentacion\%20del\%20libro\%20La\%20Habana.pdf, page consultée le 25 novembre 2017.

Ortega, Jaime (c), «'YO ERA LA CARTA'. El cardenal cubano Jaime Ortega revela el rol que desempeñó en la caída del "muro del Caribe", el histórico acercamiento entre Estados Unidos y Cuba ", Tierras de America, 4 février 2017, http://www.tierrasdeamerica.com/2017/02/04/yo-erala-carta-el-cardenal-cubano-jaime-ortega-revela-el-rol-que-desempeno-en-la-caida-del-murodel-caribe-el-historico-acercamiento-entre-estados-unidos-y-cuba/, page consultée le 25 novembre 2017.

Pape François, Discours au corps diplomatique accrédité près le Saint-Siège, 13 janvier 2014, http://w2.vatican.va/content/francesco/fr/speeches/2014/january/documents/papafrancesco_20140113_corpo-diplomatico.html, page consultée le 25 novembre 2017.

Parsons, Christi et Michael Memoli, « Obama aides discuss Vatican role in warming relations with Cuba ", Los Angeles Times, 10 avril 2015, http://www.latimes.com/world/mexico-americas/la-fgobama-cuba-20150410-story.html, page consultée le 25 novembre 2017.

Pullella, Philip, « Pope's Latin America Brains Trust Was behind Cuba Deal », Reuters, 19 décembre 2014, http://www.reuters.com/article/us-pope-cuba-mediation-idUSKBNOJX1VG20141219, page consultée le 25 novembre 2017. 
Quigley, Thomas, «The Catholic Church and Cuba's International Ties ", in Margaret Crahan (dir.), Religion, Culture and Society: The Case of Cuba, Woodrow Wilson Center Report on the Americas, $n^{\circ} 9,2003$, p. 93-102.

Reese, Thomas, « Pope Francis, like Trump, is reluctant to condemn Russia on Syria and Ukraine ", National Catholic Reporter, 8 juin 2017, https://www.ncronline.org/blogs/faith-and-justice/ pope-francis-trump-reluctant-condemn-russia-syria-and-ukraine, page consultée le 25 novembre 2017.

San Martin, Ines, " Pope Francis's Impact on Cuban, and its Church, is Already Being Felt », Crux, 15 septembre 2015, https://cruxnow.com/church/2015/09/15/pope-francis-impact-on-cubaand-its-church-is-already-being-felt/, page consultée le 25 novembre 2017.

Ticchi, Jean-Marc, « Bons offices, médiations, arbitrages dans l'activité diplomatique du SaintSiège de Léon XIII à Benoît XV », Mélanges de l'École française de Rome. Italie et Méditerranée, vol. 102, $\mathrm{n}^{\circ} 2,1993$, p. 567-612.

TV2000, Entretien avec Mgr Becciu, Substitut pour les Affaires générales de la secrétairerie d'État : « Papa, mons. Becciu: "Cuba e Usa hanno firmato disgelo davanti al Segretario di Stato Vaticano" ", TV2000, 18 septembre 2015, http://www.tv2000.it/blog/2015/09/18/papa-monsbecciu-cuba-e-usa-hanno-firmato-disgelo-davanti-al-segretario-di-stato-vaticano/, page consultée le 25 novembre 2017.

Vallely, Paul, Pope Francis, The Struggle for the Soul of Catholicism, New York, Bloomsbury, 2015.

Van Heuven, Martin à Rozanne Ridgway, « The Vatican and US policy : Saturday Reading », 25 novembre 1985, 2, 25, archives personnelles de William Wilson, Université de Georgetown, Washington, D.C.

Weigel, George, Witness to Hope: The Biography of Pope John Paul II, New York, First Cliff Street Books, 2001

Wikileaks, «Caritas International on Cuba - «Cautious Optimism » », 14 mars 2001, https:// wikileaks.org/plusd/cables/01VATICAN1436_a.html, page consultée le 25 novembre 2017.

Wikileaks, « Holy See Cautious on Cuban Dissidents », 8 novembre 2002, https://wikileaks.org/ plusd/cables/02VATICAN5471_a.html, page consultée le 25 novembre 2017.

Wikileaks, « Paya Visits Pope; FM Tauran May Reciprocate », 16 janvier 2003a, https:// wikileaks.org/plusd/cables/03VATICAN195_a.html, page consultée le 25 novembre 2017.

Wikileaks, « Holy See's Passive Approach to GOC Dissent Crackdown », 2 avril 2003b, 03VATICAN1406_a, https://wikileaks.org/plusd/cables/03VATICAN1406_a.html, page consultée le 25 novembre 2017.

Wikileaks, « Holy See Views on Cuban Transition », 10 août 2006a, 06VATICAN168, https:// wikileaks.org/plusd/cables/06VATICAN168_a.html, page consultée le 25 novembre 2017.

Wikileaks, « Scenesetter for Codel Smith », 12 octobre 2006b, 06VATICAN216, https:// wikileaks.org/plusd/cables/06VATICAN216_a.html, page consultée le 25 novembre 2017.

Wikileaks, «Holy See : DAS Madison Meeting with Deputy FM on Cuba, Nicaragua », 24 octobre 2006c, https://wikileaks.org/plusd/cables/06VATICAN225_a.html, page consultée le 25 novembre 2017

Wikileaks, «Vatican Secretary of State to Visit Cuba », 17 octobre 2007, https://wikileaks.org/ plusd/cables/07VATICAN150_a.html, page consultée le 25 novembre 2017. 
Wikileaks, «Holy See Secretary of State Talks about Cuba », 7 mars 2008, https://wikileaks.org/ plusd/cables/08VATICAN31_a.html, page consultée le 25 novembre 2017.

Wikileaks, « Vatican Hopes for Better US Cuban Ties, in Part to Rein in Chavez and his Acolytes ", 22 avril 2009a, https://www.theguardian.com/world/us-embassy-cables-documents/203608, page consultée le 25 novembre 2017.

Wikileaks, «Holy See : Scenesetter for the President's July 10 Visit », 26 juin 2009b, 09VATICAN78, https://wikileaks.org/plusd/cables/09VATICAN78_a.html, page consultée le 25 novembre 2017.

Wikileaks, « Vatican Official on Cuba Relations with EU and US », 21 janvier 2010, https:// wikileaks.org/plusd/cables/10VATICAN14_a.html, page consultée le 25 novembre 2017.

Wooden, Cindy, « Castro Declares Good Friday a Holiday », Catholic Herald, 28 mars 2012, http:// www.catholicherald.co.uk/news/2012/03/28/pope-asks-castro-to-make-good-friday-a-holiday/, page consultée le 25 novembre 2017.

\section{NOTES}

1. On citera à titre d'exemple l'arbitrage entre le Pérou et l'Équateur de 1893 et entre SaintDomingue et Haïti de 1895 sur des questions de frontières ; la médiation de 1896 entre l'Argentine et le Chili, puis entre 1900 et 1903 ; celle de 1905 entre le Chili et la Colombie ; l'arbitrage de 1906 concernant les frontières de l'Équateur et de la Colombie; et entre le Brésil et la Bolivie et le Brésil et le Pérou sur la propriété de gisements d'or (Barberini, G., 2003 : 178).

2. Sur l'Ostpolitik d'Agostino Casaroli - qui pour certains permit des avancées concrètes pour l'Église (avec la Yougoslavie, signature d'un protocole autorisant les ecclésiastiques à exercer leur activité de pasteurs et les évêques à maintenir des liens avec Rome, établissement de relations diplomatiques ; avec la Hongrie, autorisation faite au pape de nommer des évêques après approbation du gouvernement) et pour d'autres constitua un jeu de dupes pour le Vatican (lourdes concessions de sa part et non-respect des engagements pris par les gouvernements envers les diplomates catholiques) - on lira avec profit les mémoires de Mgr Casaroli (Casaroli A. , 2000) ; le chapitre «Les aléas de l'Ostpolitik », de l'ouvrage de Bernard Lecomte (Lecomte B., 2007 : 57-68), l'ouvrage The Vatican and the Red Flag et plus particulièrement les chapitres «Great Expectations and Broken Promises » et « The Small Steps of Paul VI» (Luxmoore J. et J. Babuch, 1999 : 129-153, 154-175).

3. En italique dans le texte original.

4. Wikileaks. Câbles diplomatiques envoyées par l'ambassade américaine près le Saint-Siège au Département d'État.

Voir 2006c : « Holy See : DAS Madison Meeting with Deputy FM on Cuba, Nicaragua », https:// wikileaks.org/plusd/cables/06VATICAN225_a.html ;

Ibid., 2002 : «Holy See Cautious on Cuban Dissidents », https://wikileaks.org/plusd/cables/ 02VATICAN5471_a.html;

Ibid., 2003a : « Paya Visits Pope; FM Tauran May Reciprocate », https://wikileaks.org/plusd/ cables/03VATICAN195_a.html ;

Ibid., 2003b : « Holy See's Passive Approach to GOC Dissent Crackdown », https://wikileaks.org/ plusd/cables/03VATICAN1406_a.html;

5. Ibid.,2001 : «Caritas International on Cuba - «Cautious Optimism»», https://wikileaks.org/ plusd/cables/01VATICAN1436_a.html;

6. Ibid., 2009b : « Holy See : Scenesetter for the President's July 10 Visit », https://wikileaks.org/ plusd/cables/09VATICAN78_a.html ; 
7. Ibid., 2006a : " Holy See Views on Cuban Transition », https://wikileaks.org/plusd/cables/ 06VATICAN168_a.html ;

Ibid., 2006c : « Holy See : DAS Madison Meeting with Deputy FM on Cuba, Nicaragua », https:// wikileaks.org/plusd/cables/06VATICAN225_a.html ;

Ibid., 2003b : « Holy See's Passive Approach to GOC Dissent Crackdown », 2 avril 2003b, https:// wikileaks.org/plusd/cables/03VATICAN1406_a.html ;

Ibid., 2006a : " Holy See Views on Cuban Transition », https://wikileaks.org/plusd/cables/ 06VATICAN168_a.html

8. Ibid., 2006b: "Scenesetter for Codel Smith ", https://wikileaks.org/plusd/cables/ 06VATICAN216_a.html ;

Ibid, 2006c : « Holy See : DAS Madison Meeting with Deputy FM on Cuba, Nicaragua », https:// wikileaks.org/plusd/cables/06VATICAN225_a.html ;

Ibid., 2006a : "Holy See Views on Cuban Transition ", https://wikileaks.org/plusd/cables/ 06VATICAN168_a.html ;

9. Ibid, 2002 : « Holy See Cautious on Cuban Dissidents », https://wikileaks.org/plusd/cables/ 02VATICAN5471_a.html;

10. Ibid., 2003b : " Holy See's Passive Approach to GOC Dissent Crackdown ", https:// wikileaks.org/plusd/cables/03VATICAN1406_a.html ;

11. Ibid., 2002 : " Holy See Cautious on Cuban Dissidents », https://wikileaks.org/plusd/cables/ 02VATICAN5471_a.html;

12. Ibid., 2006c : « Holy See : DAS Madison Meeting with Deputy FM on Cuba, Nicaragua », https:// wikileaks.org/plusd/cables/06VATICAN225_a.html ;

13. Cette amertume fut visible lors de son premier voyage en Pologne après la chute du Mur, en 1991, où un pape à l'air sévère mit en avant devant une population en liesse « les pièges d'une liberté coupée de toute norme morale ». Cette déception quant au tournant pris par les anciens pays du bloc de l'Est l'aurait même conduit à se mettre en retrait de la scène internationale pour se consacrer, pendant le reste de son pontificat, aux questions d'éthique et de morale (Weigel G., $2001: 642-644)$.

14. Ibid., 2006c : « Holy See : DAS Madison Meeting with Deputy FM on Cuba, Nicaragua », https:// wikileaks.org/plusd/cables/06VATICAN225_a.html ;

15. Ibid., 2009a : " Vatican Hopes for Better US Cuban Ties, in Part to Rein in Chavez and his Acolytes ", https://www.theguardian.com/world/us-embassy-cables-documents/203608 ;

16. Ibid., 2001 : «Caritas International on Cuba - «Cautious Optimism » », https://wikileaks.org/ plusd/cables/01VATICAN1436_a.html ;

17. Ibid., 2002 : « Holy See Cautious on Cuban Dissidents », https://wikileaks.org/plusd/cables/ 02VATICAN5471_a.html;

18. Ibid., 2008 : «Holy See Secretary of State Talks about Cuba », https://wikileaks.org/plusd/ cables/08VATICAN31_a.html ;

19. Ibid., 2007 : «Vatican Secretary of State to Visit Cuba », https://wikileaks.org/plusd/cables/ 07VATICAN150_a.html ;

20. Ibid., 2009.

21. Ibid., 2010 : « Vatican Official on Cuba Relations with EU and US », https://wikileaks.org/ plusd/cables/10VATICAN14_a.html ;

22. Jaime Ortega affirme avoir reçu fin 2013 une lettre de Rolando Saraff Trujillo, Cubain accusé d'espionnage par La Havane pour le compte des États-Unis emprisonné depuis près de 20 ans. Ce dernier se serait proposé comme monnaie d'échange en lieu et place d'Alan Gross, que les Américains refusent de considérer comme tel dans les négociations pour la libération des trois Cuban Five. Saraff a bien été libéré le 17 décembre 2014 (Ortega J., 2017 : 106). 
23. A ce sujet, le pape aurait dit à Mgr Ortega : «Tu n'auras pas de problème pour obtenir une entrevue avec Raul Castro, trouve le moyen d'en faire autant avec le président Obama » (Ortega J., 2017b : 3).

\section{RÉSUMÉS}

L'annonce du rôle joué par François, premier pape des Amériques, dans le rapprochement cubano-américain de décembre 2014, soulève plusieurs questions. Sollicité par les deux parties, le pape a exhorté Barack Obama et Raul Castro à surmonter l'obstacle des prisonniers politiques qui obérait la négociation et s'est porté garant de l'accord signé par les deux parties en accueillant une de leurs ultimes séances de négociation au Saint-Siège. Outre une première ébauche de la chronologie de la médiation pontificale, des motivations qui ont conduit Washington et La Havane à se tourner vers le Vatican, ainsi que de l'effet de cette médiation, cet article a pour objectif de replacer cette intervention dans le contexte plus large des priorités de la diplomatie papale, notamment vis-à-vis de Cuba, depuis le pontificat de Jean-Paul II qui fut le théâtre d'un premier dégel entre Rome et La Havane.

Le fait que l'Église catholique, devenue interlocutrice privilégiée du régime, accepte d'assurer une médiation, après des années passées à ignorer les exhortations de Washington à jouer un rôle de catalyseur de la transition démocratique, sur le modèle de la Pologne des années 1980, ne signifie pas un changement de priorités au Vatican. Si François souhaite par son action replacer le Saint-Siège sur le devant de la scène diplomatique, c'est bien pour y porter les préoccupations - défense des chrétiens menacés, maintien d'un espace pour l'exercice de la mission ecclésiale et promotion du dialogue entre États - qui sont celles de l'Église depuis la fin du XIX ${ }^{\mathrm{e}}$ siècle, moment à partir duquel elle exerce un rôle actif sur la scène internationale.

News of the role played by Francis, the first pope of the Americas, in the December 2014 rapprochement between Cuba and the United States raises a number of questions. The pope, at the request of both sides, called on Barack Obama and Raul Castro to overcome the obstacle of political prisoners which had hitherto hindered the talks. He then acted as guarantor of the agreement signed by both parties by hosting one of their final negotiating sessions at the Vatican. This article has several aims. It first purports to provide a first sketch of the papal mediation, and to explain the motivations leading Washington and Havana to turn to the Vatican, as well as the consequences of this mediation. It also aims at placing this intervention within the larger framework of the priorities of papal diplomacy - especially vis-a-vis Cuba since John Paul II's pontificate, which witnessed the first stages of a thaw between Rome and Havana.

The Roman Catholic Church, now the regime's privileged interlocutor, did agree to mediate between the two parties, after years of ignoring Washington's requests to act as a catalyst for democratic transition on the island, along the lines of the role it had played in Poland in the 1980s. Yet, this does not mean a change in Vatican priorities. While Francis, through his actions, hopes to put the Holy See back at the center of the diplomatic stage, he thereby hopes to be able to promote concerns - such as defending Christians under threat, preserving for the church a space to carry out its mission and promoting dialogue between states - which have been central to the Church since it began playing an active role in international policy in the late 19th century. 
El anuncio según el cual Francisco, el primer papa de las Américas, había tenido un papel en el acercamiento cubano-estadounidense de diciembre 2014, levanta varias preguntas. Solicitado por ambos países, el papa instó a Barack Obama y a Raúl Castro a que superen el problema de los presos políticos que obstaculizaba la negociación y garantizó el acuerdo firmado por ambas partes, acogiendo unas de sus últimas sesiones de trabajo en la Santa Sede. El objetivo de este artículo es esbozar una primera cronología de esta mediación pontifical, de las motivaciones que han empujado Washington y la Habana a pedir la ayuda del Vaticano, y de describir la consecuencias de esta mediación. Trata también de colocar esta intervención en el contexto más amplio de las prioridades de la diplomacia pontifical, especialmente hacia Cuba, desde los años de Juan Pablo II, cuyo pontificado evidenció el inicio de un deshielo entre Roma y la Habana.

El hecho que la Iglesia católica, ya el interlocutor número uno del gobierno, acepte mediar, después de años sin acceder a las demandas de Washington de actuar como catalizador hacia una transición democrática, según el modelo seguido en Polonia en los años 1980, no significa que el Vaticano haya cambiado sus prioridades. Si Francisco quiere, a través de sus acciones, volver a colocar la Santa Sede en el centro del escenario diplomático, lo hace para promocionar las preocupaciones (defensa de los cristianos amenazados, preservación de un espacio para la misión de la Iglesia, y promoción del diálogo entre Estados) que son las de la Iglesia desde que el Vaticano empezó a desarrollar un papel importante en el escenario internacional a finales del siglo XIX.

INDEX

Mots-clés : bons offices, Vatican, lettre, liberté religieuse, transition démocratique Palabras claves : buenos oficios, Vaticano, carta, libertad religiosa, transición democrática Keywords : good offices, Vatican, letter, religious freedom, democratic transition

\section{AUTEUR}

\section{MARIE GAYTE}

Marie Gayte enseigne la civilisation des Etats-Unis à l'université de Toulon. Spécialiste des relations entre religion et politique aux Etats-Unis, elle s'intéresse tout particulièrement au rôle des catholiques, clercs et laïcs, dans la politique intérieure américaine. Son travail porte également sur la dimension religieuse dans la diplomatie de ce pays. Elle vient de publier avec Blandine Chelini-Pont et Mark Rozell un ouvrage intitulé Catholics and US Politics After the 2016 Elections. Understanding the "Swing Vote aux éditions Palgrave Mac Millan. 